\title{
Simulations of the vortex in the Dellenback abrupt expansion, resembling a hydro turbine draft tube operating at part-load
}

\author{
H Nilsson \\ Chalmers University of Technology, SE-412 96 Gothenburg, Sweden \\ E-mail: hani@chalmers.se
}

\begin{abstract}
This work presents an OpenFOAM case-study, based on the experimental studies of the swirling flow in the abrupt expansion by Dellenback et al.[1]. The case yields similar flow conditions as those of a helical vortex rope in a hydro turbine draft tube working at part-load. The case-study is set up similar to the ERCOFTAC Conical Diffuser and Centrifugal Pump OpenFOAM case-studies [2,3], making all the files available and the results fully reproducable using OpenSource software. The mesh generation is done using $\mathrm{m} 4$ scripting and the OpenFOAM built-in blockMesh mesh generator. The swirling inlet boundary condition is specified as an axi-symmetric profile. The outlet boundary condition uses the zeroGradient condition for all variables except for the pressure, which uses the fixed mean value boundary condition. The wall static pressure is probed at a number of locations during the simulations, and post-processing of the time-averaged solution is done using the OpenFOAM sample utility. Gnuplot scripts are provided for plotting the results. The computational results are compared to one of the operating conditions studied by Dellenback, and measurements for all the experimentally studied operating conditions are available in the case-study.

Results from five cases are here presented, based on the kEpsilon model, the kOmegaSST model, and a filtered version of the same kOmegaSST model, named kOmegaSSTF [4,5]. Two different inlet boundary conditions are evaluated. It is shown that kEpsilon and kOmegaSST give steady solutions, while kOmegaSSTF gives a highly unsteady solution. The time-averaged solution of the kOmegaSSTF model is much more accurate than the other models. The kEpsilon and kOmegaSST models are thus unable to accurately model the effect of the largescale unsteadiness, while kOmegaSSTF resolves those scales and models only the smaller scales. The use of two different boundary conditions shows that the boundary conditions are more important than the choice between kEpsilon and kOmegaSST, for the results just after the abrupt expansion.
\end{abstract}

\section{Introduction}

The turbulent swirling flow in an abrupt expansion of 1:1.94 was experimentally investigated by Dellenback et al. [1]. The geometry is illustrated in figure 1, including the full extent of the computational domain of the present work. The inlet is located at the smaller diameter, where either a pure axial, or a swirling flow at different swirl and Reynolds numbers was imposed during the experiments.

Numerical studies of the Dellenback abrupt expansion case have been performed by Schluter et al. [6], and Paik and Sotiropoulos [7]. Gyllenram et al. [8] validated a filtered k-omega SST model using the Dellenback Abrupt Expansion case, using the optimal filter width $(\alpha=3)$, determined by Gyllenram and Nilsson [9]. Nilsson and Gyllenram [5] implemented the kOmegaSSTF model in OpenFOAM, and validated it in the Dellenback abrupt expansion case. 


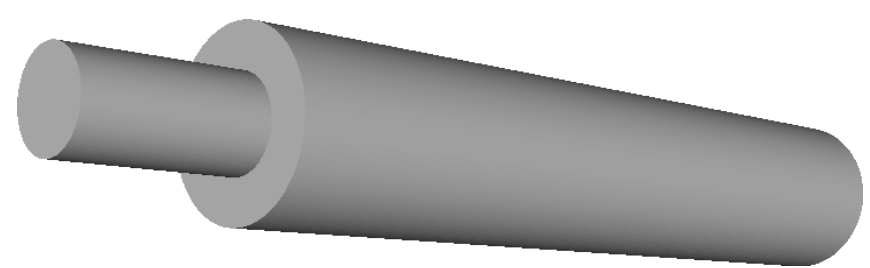

Figure 1. The Dellenback abrupt expansion geometry.

In the present work, the steady and unsteady Reynolds-Averaged Navier-Stokes equations are solved using three different turbulence models, the standard kEpsilon model, the komegaSST model, and a filtered kOmegaSST model. The near-wall region is in the present work treated using wallfunctions. The simulations are done with OpenFOAM-1.5, and can be fully reproduced since they are completely based on OpenSource software. The case-study, with descriptions, and the kOmegaSSTF turbulence model, is available at the OpenFOAM Turbulence Working Group wiki, http://openfoamwiki.net/index.php/Sig_Turbulence_/Dellenback_Abrupt_Expansion. The case-study includes complete case set-up, and automatic post-processing using the OpenFOAM utilities sample and foamLog, and plotting scripts for Gnuplot. The drawings in the present work are done with xFig, and those files are also available for editing and use in future work. Further, the case-study is accompanied with scripts for automization of the solution process and post-processing. The blockstructured mesh is parametrized using the $\mathrm{m} 4$ script language, and several mesh scripts are available.

\section{Geometry, experimental data, mesh, boundary conditions and numerical settings}

The geometry is shown in figure 1 , and the corresponding geometric data is shown in table 1 . Dellenback [1] presented 9 cases, 3 with purely axial flow, and 6 with swirl, as shown in table 2 , along the sections shown in figure 2 . At each cross-section the following data is available:

- $\quad$ Axial mean velocity profiles.

- $\quad$ Tangential mean velocity profiles.

- $\quad$ Axial RMS velocity profiles.

- $\quad$ Tangential RMS velocity profiles.

Most data sets show measurements on both sides of the centerline as a check for symmetry. The current work uses the case with $\mathrm{Re}=30,000$ and $\mathrm{S}=0.60$, for setting the inlet boundary condition at $\mathrm{Z} / \mathrm{D}=-2.00$, and for validation at $\mathrm{Z} / \mathrm{D}=0.25,0.50,0.75,1.00,1.50,2.00,3.00$, and 4.00 .

\begin{tabular}{|l|l|}
\hline Inlet diameter & $D_{\text {in }}=50.78 \mathrm{~mm}$ \\
\hline Outlet diameter & $D_{\text {out }}=98.5 \mathrm{~mm}$ \\
\hline Expansion ratio & $D_{\text {out }} / D_{\text {in }}=1.94$ \\
\hline Inlet length & $2 * D_{\text {in }}$ \\
\hline Outlet length & $10^{*} D_{\text {in }}$ \\
\hline
\end{tabular}

Table 1. Geometric data

\begin{tabular}{|l|l|}
\hline$R e=\frac{U_{b, i n} D_{i n}}{\nu}$ & $S=\left.\frac{\int_{0}^{R_{i n}} V_{\theta} V_{z} r^{2} d r}{R_{\text {in }} \int_{0}^{R_{\text {in }}} V_{z}^{2} r d r}\right|_{z / D_{\text {in }}=-2.00}$ \\
\hline 30,000 & $0.00,0.60,0.98$ \\
\hline 60,000 & $0.00,1.16$ \\
\hline 100,000 & $0.00,0.17,0.74,1.23$ \\
\hline
\end{tabular}

Table 2. Available experimental cases

The mesh block topology, shown in figure 3, is based on the one used by Gyllenram and Nilsson [4]. The mesh consists of 1,228,584 hexahedral cells. The axi-symmetric inlet axial and tangential velocities are interpolated from the experimental data, with an addition of a log-law at the wall to cover the region between the first measurement point and the wall [4], while the radial velocity is set to zero. Two different approximations of the inlet turbulence is used. The first one is the default alternative in the available case-study, yielding a turbulent to laminar viscosity ratio of $v_{\mathrm{t}} / \mathrm{v}=\mathrm{k} /(\omega v)=\mathrm{C}_{\mu} \mathrm{k}^{2} /(\varepsilon v)=16.3$, from $\mathrm{k}=3 / 2\left(\mathrm{I} \mathrm{U}_{\mathrm{b}, \mathrm{in}}\right)^{2}, \varepsilon=\mathrm{k}^{3 / 2} / 1_{\mathrm{t}}, \omega=\mathrm{k}^{1 / 2} /\left(\mathrm{C}_{\mu} \mathrm{l}_{\mathrm{t}}\right)$, where $\mathrm{U}_{\mathrm{b}, \text { in }} \approx 0.452 \mathrm{~m} / \mathrm{s}, \mathrm{I}=0.1$, $1_{\mathrm{t}}=0.1 * \mathrm{R}_{\mathrm{in}} \approx 0.0025 \mathrm{~m}$, and $\mathrm{C}_{\mu}=0.09$. The second alternative (here denoted $\mathrm{GN}$ ) is the same as was used by Gyllenram and Nilsson [4], which gives a too high viscosity ratio of $v_{t} / v=420$, using $k=I U_{b, i n}{ }^{2}$, 
$\varepsilon=\mathrm{k}^{3 / 2} / \mathrm{R}_{\text {in }}, \omega=\mathrm{k}^{1 / 2} /\left(\mathrm{C}_{\mu} \mathrm{R}_{\text {in }}\right)$, where $\mathrm{U}_{\mathrm{b}, \text { in }} \approx 0.452 \mathrm{~m} / \mathrm{s}, \mathrm{I}=0.1, \mathrm{R}_{\mathrm{in}} \approx 0.025 \mathrm{~m}$, and $\mathrm{C}_{\mu}=0.09$. Wall functions are used at the walls. At the outlet, a zero gradient boundary condition is used for all variables except for the pressure, which uses a fixed mean value of zero.

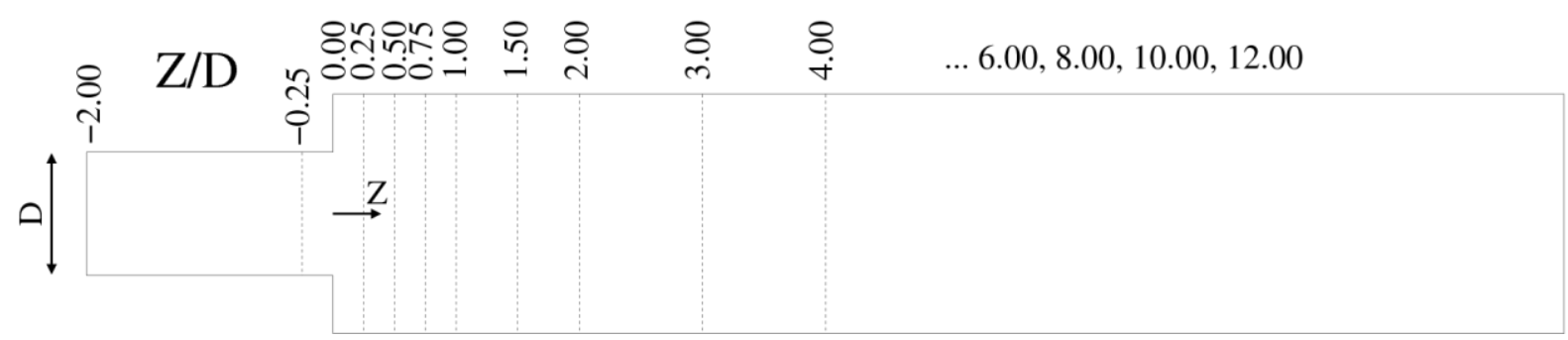

Figure 2 Measurement cross-sections. Numbers refer to $\mathrm{Z} / \mathrm{D}$, where $\mathrm{D}$ is the inlet diameter, and $\mathrm{Z}=0$ at the abrupt expansion. Sections $\mathrm{Z} / \mathrm{D}=6.00-12.00$ are not visualized by dotted lines.
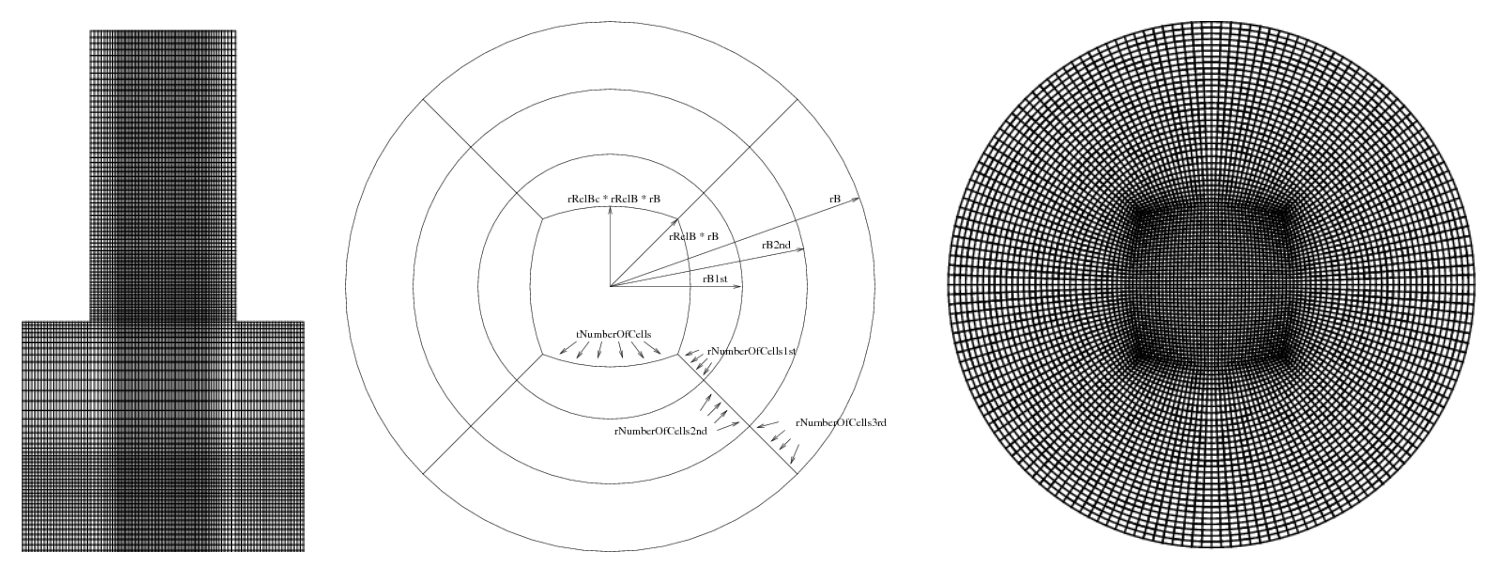

Figure 3. Block topology, $\mathrm{m} 4$ parametrization, and computational mesh.

The inner O-grid corresponds to the inlet pipe.

The pressure-velocity coupling is handled using the SIMPLE algorithm. The convection terms are discretized using the second-order linear-upwind scheme. The time term in the unsteady simulation is discretized using the second-order Crank-Nicholson scheme. The transient kOmegaSSTF simulation was run between $0-16.8 \mathrm{~s}$, averaging the values from $9.358 \mathrm{~s}$.

\section{The kOmegaSSTF turbulence model}

The kOmegaSSTF turbulence model was developed by Gyllenram and Nilsson [4]. It is a hybrid model, which limits the modeled turbulence scales. The upper limit to the modeled length scale is given by $\Delta_{\mathrm{f}}=\alpha \max \left\{|\mathbf{U}| \delta_{\mathrm{t}}, \Delta^{1 / 3}\right\}$, where $\alpha>1$ relates the length scale to the local cell size. Gyllenram and Nilsson [9] found that a value of $\alpha=3$ yields the best results, allowing unsteady vortical structures to develop, while preserving the benefits of the underlying turbulence model where needed. This value corresponds to a maximum modeled length scale of 3 cells in each direction, allowing scales that are larger than that to be resolved by the simulation. The filter is applied only on the turbulence viscosity, by multiplying it by $\left(\min \left(\mathrm{L}_{\mathrm{t}}, \Delta_{\mathrm{f}}\right) / \mathrm{L}_{\mathrm{t}}\right)^{4 / 3}$, where $\mathrm{L}_{\mathrm{t}}=\mathrm{k}^{1 / 2} /\left(\beta^{*} \omega\right)$ is the local modeled turbulence length scale from the underlying turbulence model. The rest of the system is automatically influenced by the filter, due to the apperance of $v_{t}$ in the transport equations. The base for the implementation is here the k- $\omega$ SST model by Menter and Esch [10], but the filter may also be applied to other turbulence models. 


\section{Results}

First of all it can be noted that all cases except the kOmegaSSTF model case were unable to capture the unsteadiness due to the helical vortex, also when run in transient mode. Figures 4-6 show contour plots of the results at the mid-plane. Figure 4 shows the turbulent kinetic energy. It can be observed that the results from the kEpsilon and kOmegaSST behave qualitiatively similar, with an exaggerated turbulent kinetic energy production in the stagnation region at the sudden expansion. These elevated values are the reason for the dampening of the unsteady behaviour, yielding steady results. The results differ significantly in the inlet pipe depending on which turbulence inlet boundary condition is used. The differences in the inlet pipe also influence the modelled turbulent kinetic energy downstream the expansion. The time-averaged results of the kOmegaSSTF, on the other hand, yields much lower levels of turbulent kinetic energy throughout the domain. Those numerical results turn unsteady, resolving the large helical vortex structure.

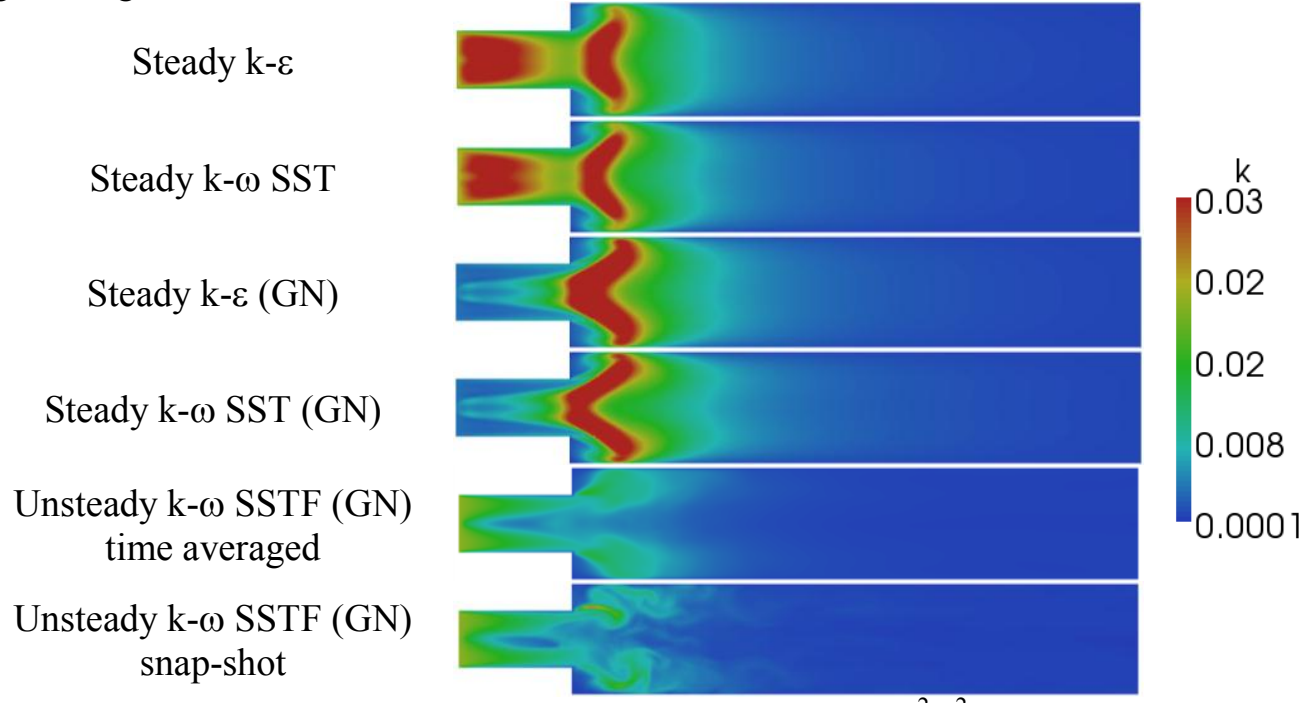

Figure 4. Turbulent kinetic energy $\left[\mathrm{m}^{2} / \mathrm{s}^{2}\right]$

Figure 5 shows the static pressure distribution at mid-plane. Again, the results from kEpsilon and kOmegaSST are quite similar, while there is an effect related to the inlet boundary condition. Although the GN boundary condition has a too high turbulent to laminar viscosity ratio, the flow in the inlet pipe seems to develop earlier than the cases with more reasonable values at the inlet. Turning to the kOmegaSSTF case, the flow is well developed already at the inlet, and the central vortex is much more pronounced with a thinner region of lower static pressure. The snap-shot reveals an unsteady vortex breakdown just after the abrupt expansion, followed by a a quasi axi-symmetric vortex further downstream.

Figure 6 shows the velocity magnitude distribution at mid-plane. Again, the kEpsilon and kOmega models give similar results, and the GN inlet boundary condition yields earlier developed flow in the inlet pipe. The snapshot of the unsteady results shows resolved velocity fluctuations due to the transportation of turbulent vortices.

Figures 7 and 8 compares the numerical axial and tangential velocity profiles with the experimental data at cross-section $\mathrm{Z} / \mathrm{D}=0.25,0.50,0.75,1.00,1.50,2.00,3.00$, and 4.00 . The time-average of the unsteady kOmegaSSTF simulation is presented. Just after the abrupt expansion the results are ordered in three groups, where two groups are due to the inlet boundary condition. Thus, the inlet boundary condition is more important at this point than the turbulence model, as long as regular RANS models are used. The kOmegaSSTF model on the other hand, being a hybrid model, reduced the influence of the turbulence modelling and let the physics in the governing equations play out their role. The result is that the velocity profiles correspond much better to the experimental results throughout the domain. It can be concluded that the inlet boundary condition influences the results in the inlet pipe region, of 
the regular RANS simulations, in a way so that the vortex coming to the abrupt expansion is not similar to that during the experiments. The hybrid kOmegaSSTF model, on the other hand, seems to better represent the development through the inlet pipe although that is not the region where the benefits from that model is greatest. Following the flow further downstream, the influence of the RANS models start to dominate over the inlet boundary condition, reducing the distinct similarities between the simulations with the same inlet boundary condition. It can be seen that the vortex of the RANS results spread out too much, while the hybrid model keeps the integrity of the vortex much longer.

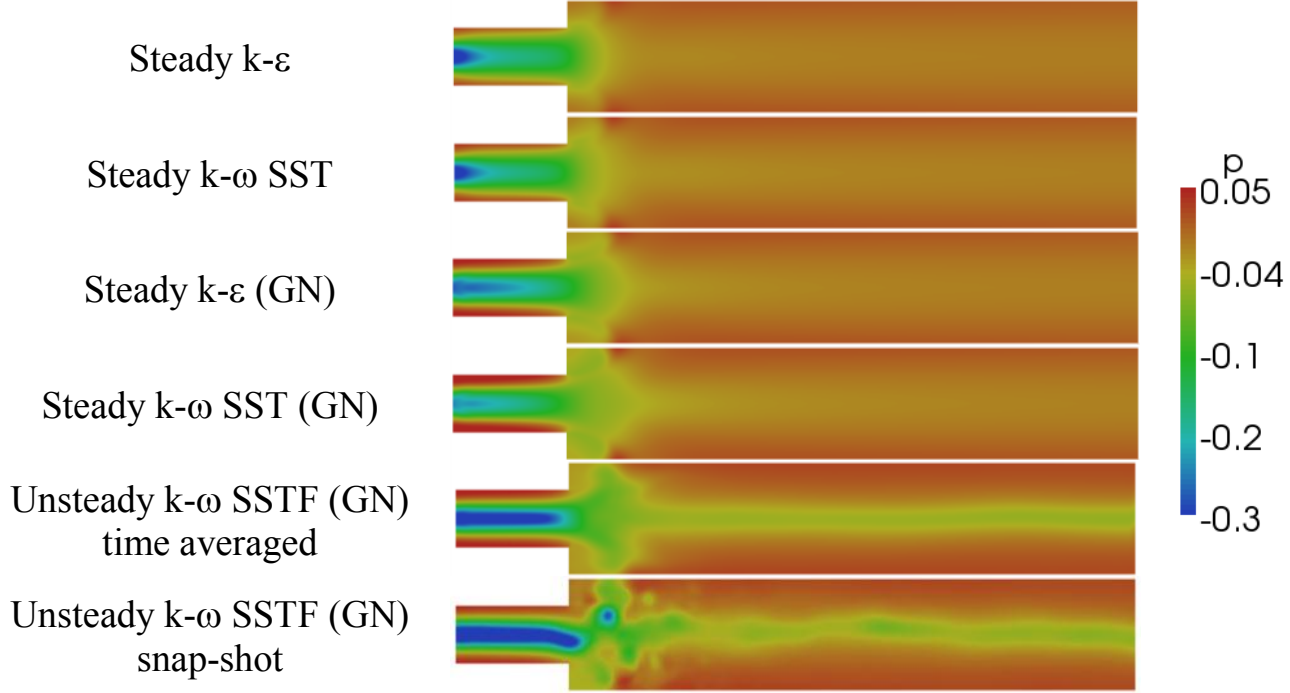

Figure 5. Static pressure, $\left(\mathrm{p}-\mathrm{p}_{\mathrm{ref}}\right) / \rho\left[\mathrm{m}^{2} / \mathrm{s}^{2}\right]$

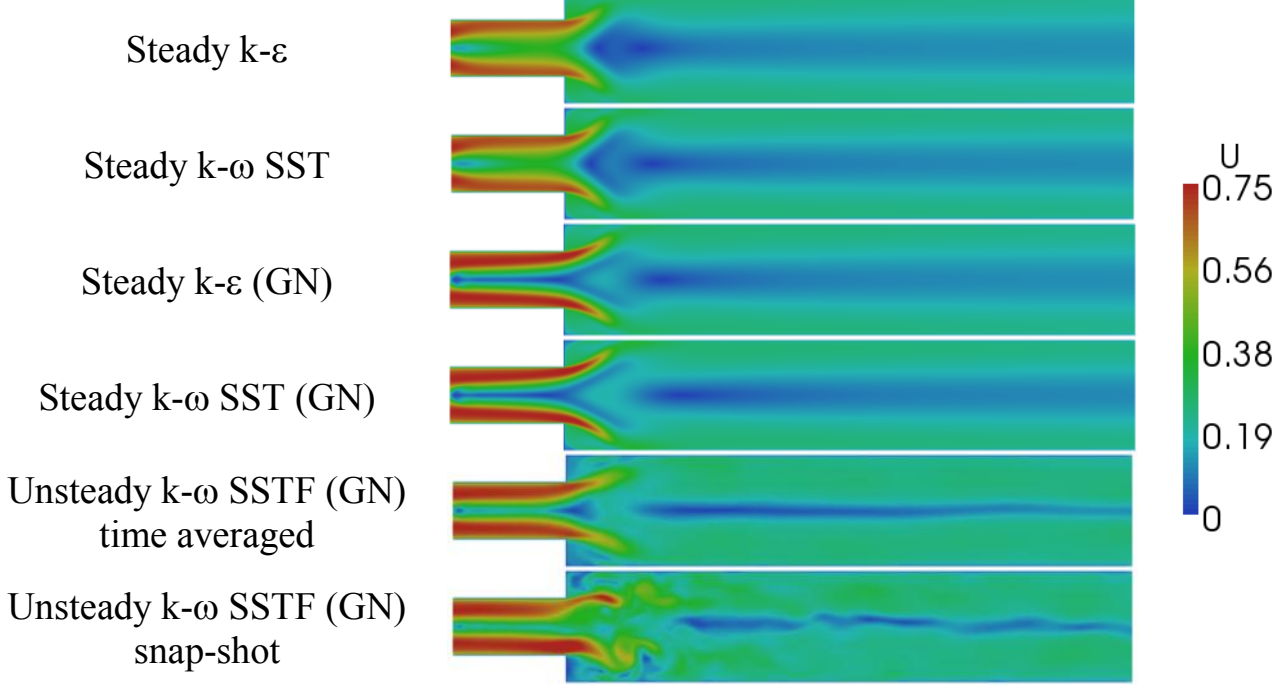

Figure 6. Velocity magnitude $[\mathrm{m} / \mathrm{s}]$

\section{Conclusions}

The main conclusion of the present work is that it is necessary to resolve the large-scale unsteadiness that occurs from helical vortex ropes, in order to correctly capture the mean velocity profiles after a vortex breakdown. The regular RANS models were not developed for modelling that kind of largescale structures, that should in fact not be seen as turbulence, and should thus be resolved rather than modelled by a turbulence model. 

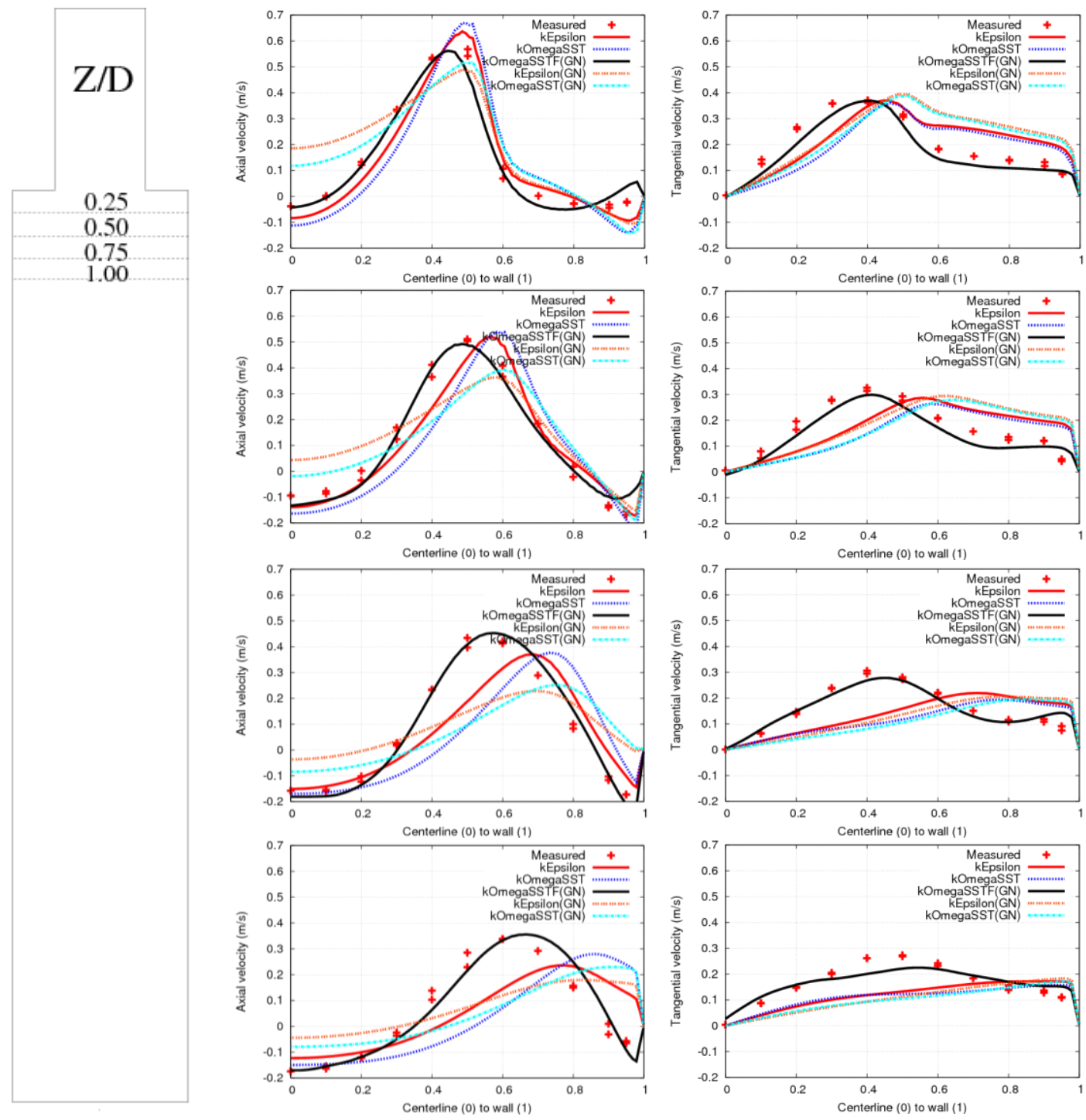

Figure 7. Comparison between numerical axial and tangential velocity distribution with the experimental data at (from top to bottom) $\mathrm{Z} / \mathrm{D}=0.25,0.50,0.75$ and 1.00 . 

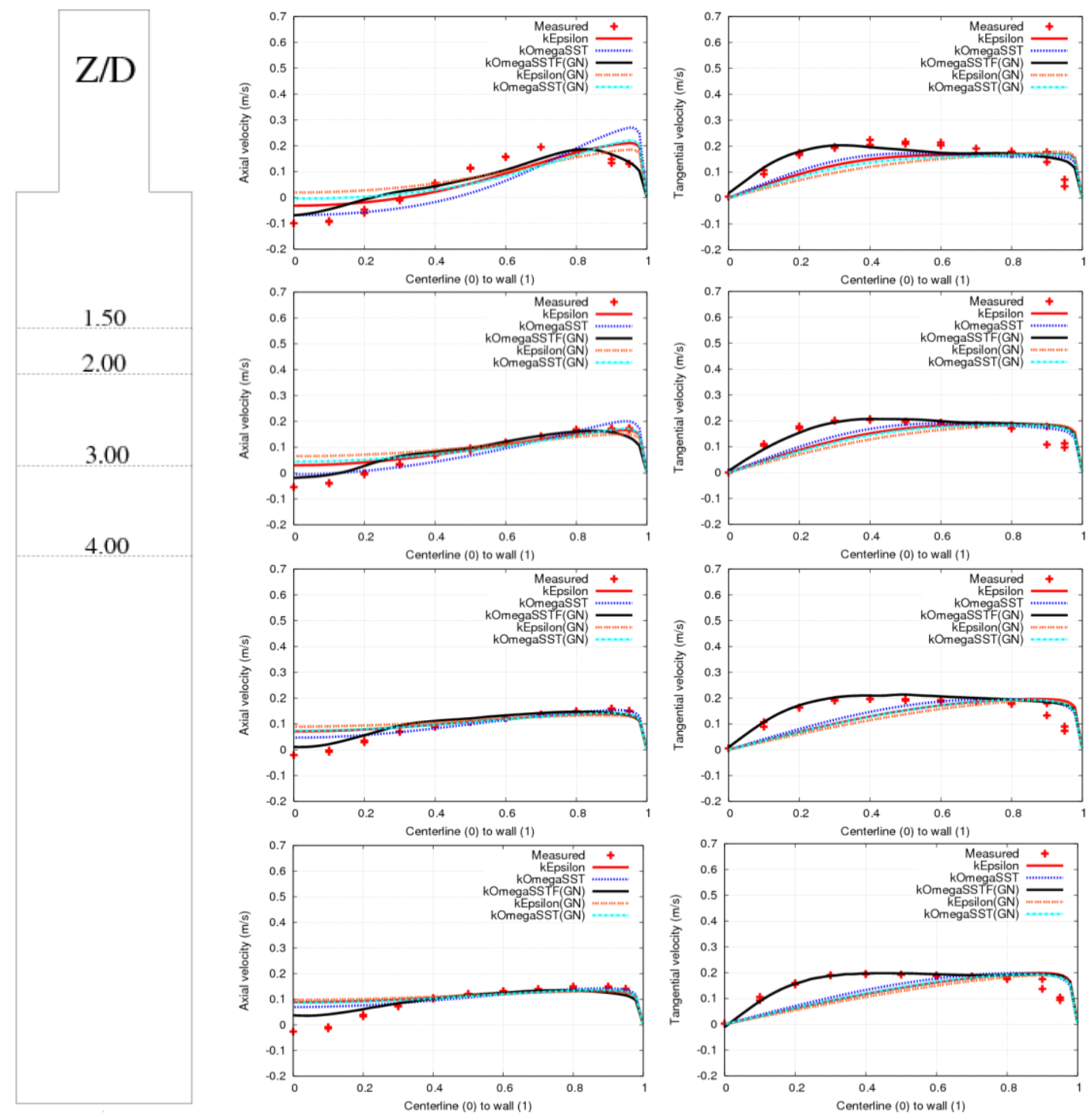

Figure 8. Comparison between numerical axial and tangential velocity distribution with the experimental data at (from top to bottom) $\mathrm{Z} / \mathrm{D}=1.50,2.00,3.00$ and 4.00 .

\section{Acknowledgements}

The research presented in this work was carried out as a part of the Swedish Hydropower Centre (SVC). SVC has been established by the Swedish Energy Agency, Elforsk and Svenska Kraftnät together with Luleå University of Technology, The Royal Institute of Technology, Chalmers University of Technology and Uppsala University (www.svc.nu). The computations were performed on resources provided by the Swedish National Infrastructure for Computing (SNIC), at $\mathrm{C}^{3} \mathrm{SE}$.

\section{References}

[1] Dellenback P A, Metzger D E and Neitzel G P1987 AIAA Journal, 26(6) 669-681

[2] Nilsson N, Page M, Beaudoin M, Gschaider B and Jasak H 2008 The OpenFOAM Turbomachinery Working Group, and Conclusions from the Turbomachinery Session of the Third OpenFOAM Workshop 24th IAHR Symposium on Hydraulic Machinery and Systems (Foz Do Iguassu, Brazil, 27-31 October 2008)

[3] Petit O, Page M, Beaudoin M and Nilsson H 2009 The ERCOFTAC centrifugal pump OpenFOAM case study 3rd IAHR International Meeting of the Workgroup on Cavitation and 
Dynamic Problems in Hydraulic Machinery and Systems (Brno, Czech Republic, 14-16 October 2009)

[4] Gyllenram W and Nilsson H 2008 J. Fluids Eng. 2008 130(5) p 051401

[5] Nilsson H and Gyllenram W 2007 Experiences with OpenFOAM for water turbine applications, Proc. of the 1st OpenFOAM International Conference (Old Windsor, United Kingdom, 26-27 November 2007)

[6] Schluter J U Pitsch H and Moin P 2004 AIAA Journal 42(3) 478-484

[7] Paik J and Sotiropoulos F 2010 Int. J. Heat and Fluid Flow 31 390-400

[8] Gyllenram W Nilsson H and Davidson L 2006 Large eddy simulation of turbulent swirling flow through a sudden expansion Proc. 23th IAHR Symposium (Yokohama,Japan, October 2006)

[9] Gyllenram W and Nilssson H 2006 Very Large eddy simulation of draft tube flow Proc. 23th IAHR Symposium (Yokohama,Japan, October 2006)

[10] Menter F and Esch T 2001 Elements of industrial heat transfer prediction Proc. 16th Brazilian congress of mechanical engineering (COBEM), pp 117-127. 